\title{
L'INTERNATIONALE ET LA PROPAGANDE : LE POINT DE VUE SITUÉ DE LA SFIO (1908-1914)
}

Vincent Chambarlhac

Société d'études jaurésiennes | « Cahiers Jaurès »

2014/2 N²12-213 | pages 79 à 90

ISSN 1268-5399

Article disponible en ligne à l'adresse :

https://www.cairn.info/revue-cahiers-jaures-2014-2-page-79.htm

\section{Pour citer cet article :}

Vincent Chambarlhac, «L'Internationale et la propagande : le point de vue situé de la SFIO (1908-1914) », Cahiers Jaurès 2014/2 (N²12-213), p. 79-90.

Distribution électronique Cairn.info pour Société d'études jaurésiennes.

(C) Société d'études jaurésiennes. Tous droits réservés pour tous pays.

La reproduction ou représentation de cet article, notamment par photocopie, n'est autorisée que dans les limites des conditions générales d'utilisation du site ou, le cas échéant, des conditions générales de la licence souscrite par votre établissement. Toute autre reproduction ou représentation, en tout ou partie, sous quelque forme et de quelque manière que ce soit, est interdite sauf accord préalable et écrit de l'éditeur, en dehors des cas prévus par la législation en vigueur en France. Il est précisé que son stockage dans une base de données est également interdit. 


\title{
L'Internationale et la propagande : le point de vue situé de la SFIO (1908-1914)
}

\author{
par Vincent Chambarlhac
}

Quels obstacles empêchent la constitution d'un espace public propre aux socialistes européens, dépassant les relations internationales ? Entendre ce questionnement générique en regard de "L'Internationale de la propagande » semble plus compliqué qu'il n'y parait. D’une part, la dynamique même de constitution d'un espace public ${ }^{1}$ autonome ne peut, du point de vue de la propagande, faire l'économie d'une territorialisation des pratiques des socialistes en la matière. La deuxième Internationale semble déterritorialisée, tout du moins n'est-elle d'aucun lieu précis, bien que dotée depuis le congrès de Paris en 1900 d'un " organisme central permanent ", fixé à Bruxelles. D'aucuns lieux pourtant, puisque les congrès sont tournants, quoique alors systématiquement européens. Cette remarque se complique du statut même de l'Internationale, questionnée au titre du concept d'espace public. Préfaçant le volume VI de l'Encyclopédie socialiste, syndicale et coopérative rédigé par Jean Longuet, Adéodat Compère-Morel évoque un " mouvement socialiste international » qui pourrait constituer un "grand parti ». La

1. La notion même d'espace public fait référence aux travaux de Jürgen HABERMAS, L'espace public. Archéologie de la publicité comme dimension constitutive de la société bourgeoise, Paris, Payot, 1998. 
question territoriale surgit rapidement dans cette dynamique conditionnelle. Le volume de Jean Longuet renseigne l'Internationale par l'histoire des sections nationales ${ }^{2}$ : la somme de celles-ci serait l'Internationale, uniquement définie par la litanie de ses congrès et résolutions. Il n'est point encore exactement d'espace public autonome des socialistes européen, sinon dans ce court temps des congrès; le bureau archivant le travail des sections nationales n'implique aucune dynamique véritable. Cet espace autonome, supposé donné par l'Internationale, participe de l'ellipse dans le temps quotidien de la propagande socialiste. Il constitue un horizon de travail, lequel comme tout point de vue demande donc d'être situé. Compère-Morel l'affirme implicitement : «Longuet a eu raison, dans ses monographies, de donner le plus de détails possibles sur la façon dont nos amis de l'Internationale entendent mener l'action parlementaire et extra-parlementaire, politique, syndicale et coopérative contre leur propre bourgeoisie ${ }^{3}$. "

Le cadre monographique s'impose pour les contemporains afin de se saisir de l'Internationale ; il pèse sur le chercheur, même si le questionnement, décentré sur la propagande et l'espace public, induit l'hypothèse d'un "agir communicationnel " socialiste et international. À sa manière, Jean Longuet valide cette dernière, évoquant pour se saisir d'une communauté de pensées et d'idées des socialistes européens, une " catholicité du socialisme ", qu'il précise ainsi : "L'observateur superficiel s'arrêtera à des différences ethniques secondaires, il sera frappé des tempéraments divers et des conditions particulières de la bataille socialiste dans telle ou telle partie du monde. Mais, celui qui ira plus au fond des choses, et s'efforcera de pénétrer la pensée intime des militants de tous les pays, leurs préoccupations doctrinales les plus essentielles, leurs controverses de tactique les plus courantes, ne pourra pas ne pas être profondément frappé par l'unité profonde, essentielle du socialisme universel ${ }^{4} »$.

2. Gilles Candar effectue une lecture serrée de ce volume : Gilles CANDAR, Jean Longuet. Un internationaliste à l'épreuve de l'histoire, Rennes, Presses universitaires de Rennes, 2007, pp. 99-107.

3. Jean LONGUET, Le mouvement socialiste international, in Adéodat COMPÈREMOREL (dir.), L'encyclopédie socialiste, syndicale et coopérative de l'Internationale ouvrière, Paris, Quillet, 1913, p. 12.

4. Ibid., p. 6. 
L'Internationale, comprise comme espace commun aux socialistes européens, participe de cette " catholicité », laquelle implique une égale Weltanschauung, une égale croyance et un répertoire commun de doctrines, de gestes, de rites, dont le marxisme constituerait le lexique. Le socle de cette " catholicité " procède du travail propagandiste qui confere un sens socialiste au mouvement des sociétés, "convertit " le militant. Ce travail aux finalités internationalistes se déploie contre une bourgeoisie nationale, dans des espaces publics nationaux que l'Internationale, comme sphère, se propose de dépasser. Le cadre monographique s'impose dans cette tension, même si, in fine, la concrétisation des efforts propagandistes suppose son dépassement. Sans le mouvement, le but n'est rien pourrait-on écrire, paraphrasant Eduard Bernstein.

À partir d'un moment éditorial de la SFIO entre le congrès de Toulouse en 1908 et août 1914, il apparaît possible de cerner les contours de " l'Internationale de la propagande ", d'envisager comment l'Internationale - et l'international - se déploie dans le registre propagandiste de la SFIO. Trois focales ponctuent cette interrogation : celle d'une figure rhétorique, "La France socialiste ", celle d'une courte interrogation du massif d'imprimés produits sur l'Internationale, ensuite, celle enfin, au ras de la question d'un ethos socialiste commun, de la dissémination du marxisme en France comme lexique de l'Internationale.

\section{La « France socialiste »}

En 1913, paraît Petit Pierre sera socialiste, illustré par Paul Grados, écrit par Jean Lorris, également directeur-propagateur de L'Encyclopédie socialiste. L'ouvrage a la forme d'un manuel scolaire. Il vise, autour de la figure centrale de Petit Pierre, à une éducation socialiste des plus jeunes. Sa visée pédagogique participe d'une modernité propagandiste passant par les registres étroitement intriqués du livre d'école, de la fiction, des lectures conseillées et des abécédaires ${ }^{5}$. Petit Pierre sera socialiste est en fait la conclusion d'un pari éditorial initié par Jean Lorris en 1908 : il s'agissait, à partir de dix brochures au mimétisme scolaire affirmé, d'éduquer,

5. Vincent Chambarlhac, Histoire d'un livre, Petit Pierre sera socialiste, Intervention à la journée d'étude " La gauche et les réseaux du livre ", Centre Georges Chevrier, Université de Bourgogne, Dijon, 20 mai 2010. Le texte a été publié dans Recherche socialiste, $\mathrm{n}^{\circ}$ 50-51, janvier-juin 2010. 
convertir, les ruraux au socialisme. Seule une brochure sera éditée en 1908, Les vérités de Pierre Mathurin ${ }^{6}$; les chapitres de Petit Pierre sera socialiste reprennent, en 1913, le plan de publication de 1908. Le contexte de 1908 importe. À la suite du congrès de Toulouse qui parachève l'unité par la synthèse ${ }^{7}$, le travail de la SFIO n'est plus de faire tenir ensemble les sectes socialistes, puisque les tendances qui en sont les héritières s'affaiblissent, mais de conquérir les terroirs par la propagande, d'élargir l'assiette du socialisme français. L'action propagandiste mute alors de manière décisive. Outre les tournées des leaders propagandistes, les brochures éternisant l'éphémère discours des tribuns, les congrès socialistes qui se suivent mettent l'accent sur la propagande écrite, sur la question de la librairie, de sa diffusion. L'Encyclopédie socialiste, initiée en 1911 par une équipe constituée autour d'Adéodat Compère-Morel ressort à ce mouvement, où l'on renseigne l'idée socialiste comme système ${ }^{8}$. Petit Pierre sera socialiste s'y arrime, par la personnalité de Jean Lorris, comme par sa forme, dérivée du manuel scolaire, en miroir de l'Encyclopédie. Il prend pour modèle Le tour de France par deux enfants, mais la progression spatiale que la titulature des chapitres donne à voir ignore le cadre national. Elle initie le lecteur au socialisme au village, débrouille la délicate question de la constitution d'une section, rencontre une grève de gueules noires et culmine par un voyage d'étude, organisé par L'Humanité, au siège du Parti Ouvrier Belge et du bureau de l'Internationale, la Maison du Peuple de Bruxelles. La forme didactique de l'ouvrage condense le rapport propagandiste à l'Internationale. Celle-ci est à la fois clé de voûte de la formation militante, horizon militant tramée par les journaux, les brochures (le voyage est organisé par L'Humanité) et ressource pour l'action (le voyage est d'étude). Avers de ce mouvement, l'Internationale se comprend dans un étroit rapport à la situation du socialisme français. Cette dialectique a comme charnière la figure de la "France socialiste".

6. V. Chambarlhac, «Ce n'est pas l'auteur qui parle. Un pari éditorial de la SFIO en 1908 ", in Laurence GIAVARINI (dir.), Pouvoir des formes, écriture des normes, Dijon, EUD, À paraître en 2014.

7. Alain Bergounioux, Éloge de la réforme. Discours de Jean Jaurès au congrès de Toulouse en 1908, Note " histoire et mémoire ", ${ }^{\circ} 9$, Paris, Fondation Jean-Jaurès, septembre 2008.

8. V. Chambarlhac, "L'Encyclopédie socialiste, une forme singulière pour une cause politique ?", Genèses, 57, décembre 2004, pp. 4-22. 
La première occurrence éditoriale du terme doit être attribuée, en 1886, à Gabriel Terrail, qui, sous le pseudonyme de Mermeix, donne, pour F. Fetscherin et Chuit éditeurs, un ouvrage titré La France socialiste, notes d'histoire contemporaine? . Sous la plume de cet ex-boulangiste publiciste, le titre s'entend comme un clin d'œil au best seller contemporain, La France juive d'Édouard Drumont (1886). La Revue socialiste, qui le chronique alors, note que l'auteur s'est fourvoyé, relevant « qu'en voulant tracer un tableau de la France socialiste, il n'a donné qu'une esquisse de la France marxiste. Il a pris la partie pour le tout ${ }^{10}$ ". La remarque participe de la manière dont les premiers congrès de la deuxième Internationale définissent l'action socialiste face à l'anarchisme, au syndicalisme, au réformisme opportuniste. Un travail de délimitation s'effectue alors, la définition du socialisme s'évide ; à sa manière, arpentant une "France socialiste ", Mermeix montre ce travail en cours.

Quand elle ressurgit, autour de 1908, la " France socialiste " devient un trope propagandiste, dont l'implicite est évidemment marxiste. La métaphore articule le local, le national à l'international. Au ras du militant, l'expression inscrit l'action socialiste dans l'optique de dépassement de la République par la Sociale, comme dans l'optique de la conquête du pouvoir et de la réforme. La « France socialiste » est l'un des ressorts propagandistes du grand récit socialiste sous la II ${ }^{e}$ Internationale. Elle est la figure de l'entreprise de la société française par la SFIO, patente depuis 1908. Par ce trope, dans le registre de l'Internationale, cette entreprise se reconnaît comme l'une des composantes de la civilisation socialiste à venir. Au présent, elle en dessine les conditions de possibilités tout en se conjuguant également dans les brochures propagandistes au futur : elle serait alors l'exacte traduction nationale du dépassement du capitalisme par le socialisme ${ }^{11}$. La "France socialiste» est l'une des conditions d'un espace commun aux socialismes européens, comme la « Belgique socialiste », l'Allemagne, l'Italie, l'Angleterre, toutes affublées, peu ou prou, du même épithète.

9. Mermeix, La France socialiste. Notes d'histoire contemporaine, Paris, F. Fetscherin et Chuit Editeurs, 1886.

10. G[ustave] R[OUAneT], "La France socialiste de Mermeix », La Revue socialiste, 19, juillet 1886, p. 669.

11. Marc Angenot, L'utopie collectiviste. Le grand récit socialiste sous la IIe Internationale, Paris, PUF, 1993. 
$\mathrm{La}$ " France socialiste " représente ainsi un biais propagandiste par quoi l'Internationale, comme anticipation d'un espace commun s'esquisse. Cet espace commun est, au niveau européen, en devenir. Il s'agit par l'examen de chaque section nationale d'en percevoir les contours dans une forme nationale, afin que la somme de ces observations fasse sens. On mesure ici l'une des apories de l'Internationale de la propagande : hormis quelques slogans - "prolétaires de tous les pays unissezvous? "-, il lui faut décliner nationalement chaque progrès socialiste. Avant que d'être le " grand parti " des travailleurs, l'Internationale est mouvement. À ce point de l'analyse, le biais propagandiste qu'est la « France socialiste " participe, contre la croyance en l'imminence d'une solution révolutionnaire, d'une pragmatique.

\section{L'Internationale de la propagande et l'imprimé}

La possibilité d'un espace commun propre aux socialistes européens suppose, pour chaque militant, au-delà de la logique de la « France socialiste » qui fait suture avec l'Internationale, de se familiariser avec celle-ci, sinon de s'en approprier les codes, les logiques. Dans l'ordre propagandiste des socialistes français, la question de l'appropriation de ses contenus paraît complexe, hiérarchisée. Deux logiques trament cette question. La première tient à ce que l'expression d'un recours à l'Internationale comme espace commun se déploie dans la société française ; la seconde, davantage arrimée à l'écrit, se duplique en interrogations parallèles : qu'écrit-on, que traduit-on, pour qui selon quels canaux de diffusion?

À hauteur de militants, des gestes, des occasions, valent monstration de la conscience d'appartenir à un espace commun, distincts des déclinaisons nationales. L'Internationale, hymne officiel de la deuxième Internationale (1892), l'illustre. Elle clôt les réunions, sonorise les cortèges, les fêtes ouvrières. Elle se décline en Internationale viticole (1907), en Internationale du beurre (1911) contre la vie chère... Le chant entonné lors des premiers Mai vaut expression unitaire, symbolise un lien qui dépasse la question nationale. Pour Jaurès « L'Internationale n’a été que la suite prolétarienne de la Marseillaise " ${ }^{12}$, et la possibilité d'un espace commun qui se donne fugacement lors du chant entonné confesse donc

12. Jean Jaurès cité par Marc FERRO, L'Internationale. Histoire d'un chant de Pottier et Degeypter, Paris, Noesis, 1996, p. 46. 
que celui-ci est prolétarien. Classiquement, on conclura que cet espace oppositionnel ${ }^{13}$, distinct de l'opinion publique bourgeoise, se donne par la classe, fugacement, dans l'événement. Une subjectivité (révolutionnaire, socialiste, ouvrière...) s'exprime, ici par le chant, ailleurs par la manifestation, contre l'ordinaire de la vie publique dont elle se démarque en affirmant son internationalisme. On retrouve cette même fugacité lors de la manifestation Ferrer en 1909, lors des manifestations de 1912 contre la guerre. Pour autant, si ces gestes participent du répertoire de l'Internationale de la propagande, ils peinent à en circonscrire le contenu, renseignent peu - sinon jamais - l'historien sur les conditions d'appropriation par le militant ou le sympathisant socialiste d'un sens propre à l'Internationale par quoi une subjectivité socialiste s'informe. Le recours aux massifs d'écrits propagandistes esquisse ces conditions.

Le concept d'espace public trouve dans l'imprimé l'une de ses formes de prédilection. En la matière, les socialistes de la Belle Époque sont prolixes. La propagande n'est pas seulement l'effet du discours du tribun, elle procède surtout de la lecture et donc de l'écrit, dimension particulièrement scrutée par les congrès de la SFIO après 1908. L'imprimé propagandiste participe alors soit du journal, soit de la brochure ; il est ensuite, pour des lecteurs plus " confirmés " celui de la revue, du livre. Dans ce dispositif, le journal de section, de ville ou de fédération, informe peu la question internationale, sinon par le marmonnement, quotidien dans L'Humanité, des actualités : peu de distance, guère de vertu propagandiste prêtée à la lecture de ces commentaires qui supposent un lecteur déjà « instruit » de ces questions. La brochure s'avère davantage pivot de ce dispositif propagandiste. Lucien Roland, pour le congrès de Nancy (1907), donne la liste des brochures dont dispose la Librairie du Parti. Le fonds, guère organisé, comprend 215 brochures, celles-ci proviennent notamment des sectes socialistes d'avant l'unité. La librairie du Parti ne s'est pas dotée d'un catalogue, la liste s'ordonne par le prix des brochures, de 5 centimes à 10 francs, aucune indication de tirage ou de date d'édition n'apparaît. Un tri grossier, permis par la seule titulature, repère 43 brochures dont les sujets participent strictement de l'horizon du mouvement socialiste international, soit $20 \%$ du fonds. $44 \%$ de cet échantillon est vendu pour une somme inférieure ou égale à 1,50 franc ; $66 \%$ pour une somme supérieure à 2,50 francs. Les chiffres trahissent un

13. Oskar Negt, L'espace public oppositionnel, Paris, Payot, 2007. 
lectorat potentiel pour ces brochures distinct du sympathisant socialiste moyen. L'horizon de l'Internationale se donne pour un lectorat sans doute plus instruit, sinon capable de consacrer une part de son pouvoir d'achat à cette question. Une lecture plus détaillée de ce fonds remarque une forte présence des ouvrages de Marx - ou leur vulgarisation-, quelques discours lors des congrès de l'Internationale (Amsterdam, 1904) auxquels il faut ajouter 2 tomes sur l'Internationale ouvrière et socialiste (2,50 francs) et quelques brochures monographiques sur le socialisme anglais, belge. L'Allemagne est représentée par des textes d'Eduard Bernstein, de Karl Kautsky ${ }^{14}$. Que conclure de cette plongée, sinon l'indice du lectorat et surtout l'absence d'une vulgate propagandiste, tout public, sur l'Internationale?

Le monde des revues, aux lectorats sans doute plus confinés, biaise ce constat, le compliquant. Le modèle revuiste des socialistes est la « revuefamille » qui regroupe et type surtout des cercles intellectuels, plus qu'elle ne dispose d'un ample lectorat. Leurs lecteurs sont souvent des intellectuels proches du parti socialiste, comme dans l'Effort libre, Documents pour le socialisme, voire La Revue socialiste... L'assise intellectuelle de ces dernières, plutôt large, procède surtout d'une mouvance vitaliste où les questions culturelles et philosophiques sont premières tramant une nouvelle culture politique ${ }^{15}$, sauf pour La Revue socialiste dont l'objet, depuis 1910, coagule le mouvement syndical, coopératif et socialiste ${ }^{16}$. Aussi, du point de vue d'une Internationale de la propagande, les revues socialistes participent davantage des logiques de réseaux et de transferts culturels. Lues ainsi, elles contribuent à la formation d'un espace autonome des socialistes européens mais circonscrits, sinon à une élite, tout du moins à un noyau intellectuel resserré dont l'influence peut sans doute davantage s'esquisser dans le rapport aux leaders socialistes, Jaurès, Albert Thomas notamment.

À ce point de l'analyse, un chiasme : l'Internationale de la propagande participe d'une logique de réseaux - en ce sens elle construit la possibilité d'un espace commun-, mais le dispositif propagandiste ne

14. Jean JAURES, Karl KAUTSKY, Socialisme et révolution française, édition et présentation de Jean-Numa DuCANGE, Paris, Demopolis, 2010.

15. Sur ce point, cf. Christophe PROCHASSON, Les intellectuels, le socialisme et la guerre, Paris, Le Seuil, 1993.

16. Madeleine RebÉRIOUX, "La Revue socialiste ", Cahiers Georges Sorel, 5, 1987, pp. 15-38. 
vise pas l'assiette la plus large du socialisme français, pour qui cet espace demeure encore largement hypothétique. La majeure partie des militants l'appréhende uniquement sous la forme nationalisée de la "France socialiste ». De facto, l'internationalisme des socialistes français est encore largement national, ce d'autant plus que cette propriété recoupe le jeu des figures de l'universel dans l'idée républicaine.

\section{Dissémination du marxisme et Internationale de la propagande}

Au cœur de ce chiasme, la question du marxisme est centrale. Elle est la condition d'un lexique commun aux socialistes européens que son extériorité au corpus républicain constitue comme lieu et borne de l'Internationale. De ce point de vue, le lexique marxiste évide la figure de la " France socialiste » de toute contingence nationale. Il faut ainsi entendre, ab origine, les réserves de La Revue socialiste.

La SFIO se réalise sur l'injonction de l'Internationale en 1905, réunissant PSdF et PSF. Au regard de sa naissance, le marxisme est dans la SFIO le langage de l'Internationale plus que celui du parti lui-même. Harvey Goldberg déduisait de ce fait le long compagnonnage de Charles Rappoport avec les guesdistes, ceux-ci se faisant l'écho de l'orthodoxie de la deuxième Internationale ${ }^{17}$. Jusqu'au congrès de Toulouse, le marxisme est un enjeu interne des luttes entre les tendances héritées des sectes socialistes. Il n'est pas là un mais des marxismes. Le pluriel implique la dissémination puisque des usages concurrents, et/ou étrangers l'un à l'autre, du marxisme se sont diffusés par capillarité dans le tissu du socialisme français. L'histoire de cette réception du marxisme outrepasse cet article ${ }^{18}$, retenons comme ligne de fuite la dispersion éditoriale de celui-ci - de la brochure ou du syllabaire guesdiste au fort volume, via l'extrait, la citation dans le journal ${ }^{19}$-, et son incomplétude. Il ne peut donc y avoir une totalité qui serait le marxisme de la SFIO puisque les

17. Harvey GoldberG, "Charles Rappoport. La crise du marxisme en France ", L'Homme et la société, 24-25, Avril / Septembre 1972, pp. 127-150.

18. C'est le travail entrepris sous la houlette de Jean-Numa DuCANGE, cf. pour de premiers jalons Cahiers d'histoire-Revue d'histoire critique, 114, janvier-mars 2011.

19. Pour une approche, encore incomplète, de cette dispersion éditoriale avant 1914, cf. sur le site de la MSH de Dijon, son portail Pandor et le corpus de la Grande Edition Marx/Engels. 
schèmes d'appropriation sont multiples, polysémiques ; l'historiographie note sobrement que ce marxisme est introuvable ${ }^{20}$. À ce constat, on opposera que la dissémination du corpus marxiste en France brise le cadre commun du lexique. Il n'est point de linéarité de la section à l'Internationale dans l'emploi de termes toujours propices aux situations de «mésentente ", aux heurts entre le " dogmatisme » des guesdistes, la souplesse du marxisme normalien, l'anti-marxisme d'Andler, le choix du révisionnisme de Bernstein pour Varenne.... Cette dissémination heurte la « catholicité » du mouvement socialiste avancée par Jean Longuet dans son volume de l'Encyclopédie consacré à l'Internationale. La notion surgit dans le contexte d'un renouveau propagandiste par l'éducation après 1909 dont Jean Longuet, «l'homme de l'Internationale » est le pivot. Il propose, s'inspirant du socialisme allemand, de viser le militant comme « homme de confiance " du socialisme français, et d'éditer pour celui-ci les œuvres majeures de Marx au nom du parti. Si une sous-commission à l'édition est créée en 1909, il faut attendre 1911 et le congrès de SaintQuentin pour que cette question ressurgisse, sans grands lendemains d'ailleurs ${ }^{21}$. Pour Longuet, cette édition participait d'une «science de la propagande ». Le terme suppose que le marxisme soit l'orthodoxie des militants de la SFIO. Deux ans plus tard, lors du congrès de Saint-Quentin, le rapport de la sous-commission à l'édition, rappelle la nécessité d'une " édition scientifique irréprochable». Celle-ci s'assortit rapidement pourtant d'une réserve. L'édition scientifique ne saurait être complète, une telle tâche relevant de la science pure quant il s'agit de s'engager dans la voie d'une science de propagande. La sous-commission se propose de « donner au public des œuvres maîtresses du grand philosophe socialiste dont la connaissance est nécessaire à la compréhension de la méthode d'action qui inspire, à l'heure actuelle, toutes les sections de l'Internationale prolétarienne ${ }^{22}$ ». Le propos est clair, le langage de l'Internationale tient aux textes de Marx, en premier chef au Livre I du Capital. La suite du programme d'édition est plus floue, évo-

20. V. ChambarlHaC, «L'orthodoxie marxiste de la SFIO, à propos d'une fausse évidence (1905-1914) ", Cahiers d'histoire-Revue d'histoire critique, 114, janvier-mars 2011, pp. 39-50.

21. G. CANDAR, Jean Longuet..., op. cit., pp. 53-54.

22. $8^{\text {e }}$ congrès du parti socialiste tenu à Saint Quentin du 16 au 19 avril 1911. Compte rendu / Parti socialiste SFIO (Congrès national; 08; 1911; Saint-Quentin) / [S.l.] : [s.n.] (1911). 
quant pêle-mêle des critiques politiques, économiques, historiques et un volume de correspondances (Marx/Engels, Lassalle, Kugelmann). Une biographie de Karl Marx, demandée à Laura Lafargue, conforte ce dispositif. Le texte de la traduction doit être revu par Alfred Bonnet et Édouard Fortin.

Dans cette configuration, la " catholicité » de l'Internationale tient à un marxisme qui se donne par la propagande, et non l'érudition scientifique. On peut ici avancer que l'Internationale de la propagande tient au marxisme, en tant qu'il engage la plénitude d'une identité socialiste dans son universalité. Le lexique marxiste engage la conformité de la SFIO à l'Internationale. C'est là plus que la recherche d'une orthodoxie sous la plume de Longuet, car la "catholicité " socialiste ne se mesure jamais à l'aune des autres sections nationales : elle en constitue la somme et l'horizon quand la propagande en est le moyen, le truchement. Ainsi, s'agit-il moins d'un "marxisme de propagande »-pour reprendre l'expression de Gilles Candar - véhiculée par les formes brèves de l'imprimé propagandiste que d'une condition sine qua non pour un véritable internationalisme.

Ramenée à l'hypothèse d'un espace commun aux socialistes européens, qui se déclinerait comme un espace public tel que théorisé par Jürgen Habermas, cette condition interroge. Elle ne peut se rapporter aux seuls massifs de publications socialistes. L'échec du projet par Jean Longuet le confesse, comme l'entreprise de systématisation et de codification menée par L'Encyclopédie socialiste, elle doit composer avec la problématique de l'édition privée, du capitalisme d'édition d'Aristide Quillet, de la Librairie Rivière, etc. À ce point, le marxisme se conjugue sous le signe de l'édition populaire - « collant » ainsi d'une certaine manière à l'esprit de la proposition de Jean Longuet- mais aussi à l'édition universitaire, apparaissant là comme un marxisme dégondé de son lien au politique, non une doctrine donc mais un auteur et une pensée sociologique, économique. L'échec alors, du point de vue de la SFIO, d'une Internationale de la propagande tient à ce qu'en tous points, ce dessein propagandiste tient au national, à la confrontation et à l'intrication avec la sphère bourgeoise républicaine. On escomptera là, qu’à l'image de l'espace public enfanté aux Lumières mais dégradé par la consommation, le rôle de l'État, au XIX ${ }^{\mathrm{e}}$ siècle, l'horizon d'une Internationale qui serait un espace public propre aux socialistes est un but chimérique, entravé qu'il est, du point de vue de la propagande, par sa nécessaire territorialisation. 
Il n'est donc pas exactement une Internationale de la propagande, mais un but et des moyens, communs aux socialistes européens mais biaisés par leur inscription dans un cadre socio-politique national. Au premier chef, l'Internationale de la propagande s'énonce sous le signe du mouvement : c'est un but, un horizon, impliquant le dépassement du capitalisme et des formes socio-politiques qui s'y associent, dont la dynamique se déploie dans cette sphère. On ne peut donc sans sourciller opiner au leitmotiv de la trahison longtemps tramé par la Troisième Internationale pour se saisir d'août 14 : cette dynamique se brise sur les nationalismes européens, alors même - au moins du point de vue de la SFIO - qu'elle tentait éditorialement de les dépasser. Ainsi, c'est dans le mouvement qu'il faut penser août 14 et ses suites. L'échec de la deuxième Internationale à empêcher la guerre dit l'incomplétude d'une construction où les coutures nationales sont encore trop visibles. La somme des mouvements socialistes ne fait pas le tout qu'est l'Internationale, que la propagande tentait de constituer. Pour autant, c'est dans la logique même de l'Internationale de la propagande que l'on peut aussi sonder l'action des socialistes dans la Grande Guerre. Pour une part des socialistes ralliés à l'Union sacrée, la guerre crée les conditions de réalisation d'une part des promesses de la "France socialiste», par la réforme, l'exercice de l'État ${ }^{23}$. L'archéologie du trope ${ }^{24}$ signale qu'il ne faut pas lire ce ralliement dans une étroite obsidionalité, la « France socialiste » est une part de la civilisation socialiste. La guerre, l'événement que sont les révolutions russes en décident autrement. En ce sens, 1917 date l'échec d'une tentative dont on fête aujourd'hui le centenaire, comme le centcinquantenaire, celui d'un espace commun de discussion, jamais réalisé depuis à l'échelle du mouvement ouvrier.

Vincent CHAMBARLHAC (uB, CGC, CNRS 5605)

23. V. Chambarlhac, " un majoritaire de guerre ", in V. Chambarlhac, Thierry HoHl, Bertrand Tillier, Léon Rosenthal. Militant, critique et historien d'art, Paris, Hermann, 2013. Et V. CHAMBARlHAC, Romain DuCOUlOMBIER (dir.), Les socialistes français et la Grande Guerre Ministres, militants, combattants de la majorité (1914-1918), Dijon, EUD, 2008.

24. On désigne par cette expression la suite des apparitions de la figure de la France socialiste, depuis l'invention de l'expression par Mermeix. 